\title{
Dynamics of the Planning Solution in the TeXtbook Model of LABor Market Search AND Matching
}

\author{
Joydeep Bhattacharya \\ Iowa State University and CentER, Tilburg University \\ Helle Bunzel* \\ Iowa State University and CentER, Tilburg University
}

May 27, 2003

\begin{abstract}
This paper takes a discrete-time adaptation of the continuous-time matching economy of Pissarides $(1990,2001)$ discussed in Ljungqvist and Sargent (2000) and computes the solution to the dynamic planning problem. The solution is shown to be completely characterized by a first-order, non-linear map which admits a unique stationary solution that is dynamically unapproachable from an arbitrary set of initial conditions. Oscillatory solutions are possible but there is no possibility of periodic solutions.
\end{abstract}

JEL Classification: J 41, J 64.

${ }^{*}$ Please address all correspondence to: Helle Bunzel, Department of Economics, 260 Heady Hall, Iowa State University, Ames, IA 50011-1070, USA. E-mail: hbunzel@iastate.edu; Ph: 1-515-294-6163; fax: 1-515-294-0221 


\section{Introduction}

This paper takes the planning solution to the standard "textbook model" of search and matching in labor markets, and shows that any kind of complex dynamics is impossible in that framework. More specifically, it takes as a starting point, Ljungqvist and Sargent's (2000; Chapter 19) discrete-time adaptation of the continuous-time matching economy described in Pissarides (1990, 2001) and computes the solution to the dynamic planning problem. The solution is shown to be completely characterized by a first-order, non-linear scalar difference equation. The main contribution of this paper is to show that a) there is a unique stationary solution, b) it is unstable in that it is dynamically unapproachable from a given set of initial conditions, and c) oscillatory solutions are possible but there is no possibility of periodic solutions. The implication is strong and clear: in an economy characterized by search and matching frictions, an omniscient social planner has no option but to pick the stationary solution straightaway.

Several papers in the literature have investigated the possibility of non-stationary solutions in search models of the labor market. The seminal papers in this area are Drazen (1988) and Diamond and Fudenberg (1989); both build on Diamond (1982) and prove the existence of stable limit cycles in a model where there are frictions in coordinating trade, and the matching technology is subject to increasing returns. More recently, Mortensen (1999) revisits the standard textbook model of search and matching in the labor market as described in Pissarides (1990) but introduces an increasing returns to scale production technology to generate multiple long-run unemployment equilibria and stable limit cycles. ${ }^{1}$ Shimer and Smith (2001) explore optimal matching policies in constant returns to scale search economies with heterogenous agents and find the possibility of non-stationarity.

The current endeavour is different from the previous literature in three important ways. First, the focus here (as in Shimer and Smith, 2001) is on the planning solution as opposed to the "decentralized" solution (the focus of the other aforementioned papers). Second, neither the production nor the matching technologies in our model exhibit any increasing returns. Finally, unlike the continuous-time framework used by Mortensen (1999) and Shimer and Smith (2001), we use the discrete-time adaptation. ${ }^{2}$ In a sense, our results suggest that merely delegating the job of coordinating labor market search activity to a planner immediately renders an economy immune to any kind of indeterminacies or endogenous

\footnotetext{
${ }^{1}$ Mortensen (1999) assumes that match productivity is an increasing function of the aggregate number of matches. This generates the needed increasing returns in production.

${ }^{2}$ The discrete-time version of the Pissarides (1990) search-and-matching story has also been employed by Merz (1995), Andolfatto (1996), Shi and Quan (1999), Cooley and Quadrini (1999), Cole and Rogerson (1999), Yuan and Li (2000), and Yashiv (2000), among others.
} 


\section{fluctuations.}

The plan for the rest of the paper is as follows. Section 2 outlines the Pissarides (1990) model of search and matching in labor markets. Section 3 contains a detailed analysis of the main difference equation alluded to earlier. Proofs of some central results are to be found in the appendices.

\section{The Model}

The model (and the notation) is based entirely on Ljungqvist and Sargent's (2000) discrete-time adaptation of the continuous-time matching economy described in Pissarides $(1990,2001)$. Let $t=0,1,2,3, \ldots$ index time. There are two types of agents: workers and firms. There is a continuum of identical workers of measure 1. These workers are all infinitely-lived, they discount the future at the rate $\beta$, and are risk-neutral. Workers potentially get matched with a firm; the result of such a match is output $y \cdot{ }^{3}$ Each firm may employ at most one worker. A firm incurs a vacancy cost of $c$ in each period when looking for a worker. A match between a worker and a firm gets dissolved with an exogenously-specified probability s. An unmatched worker is an unemployed worker; such a worker enjoys the current utility from leisure of amount $z$.

Matches are brought together by a standard matching technology connecting only unemployed job seekers with open vacancies. The number of successful matches in a period is given by $\mathcal{M}\left(u_{t}, v_{t}\right)$ where $u_{t}$ is the total measure of unemployed workers looking for jobs, and $v_{t}$ is the number of vacancies or firms looking for employees. The matching function is increasing in both arguments, concave, and homogenous of degree one. For all that we present below, we will assume a standard constant returns to scale formulation,

$$
\mathcal{M}\left(u_{t}, v_{t}\right)=A u_{t}^{\alpha} v_{t}^{1-\alpha} \quad A>0, \quad \alpha \in(0,1)
$$

where $A$ is a scale parameter. The parameter $\alpha$ is the elasticity of the matching function with respect to the measure of unemployed workers. Let $\theta_{t} \equiv v_{t} / u_{t}$ indicate the measure of labor market tightness, or the ratio of vacancies to unemployed workers. Then define $q\left(\theta_{t}\right) \equiv \mathcal{M}\left(u_{t}, v_{t}\right) / v_{t}$ denote the probability with which a vacancy gets filled at date $t$. It follows that $q(\theta)=A \theta^{-\alpha}<1$ must hold. Similarly, the probability that an unemployed worker gets matched at date $t$ is given by $\theta q(\theta)=A \theta^{1-\alpha}<1$. Finally, define $n_{t+1}$ as the total number of employed workers at the start of $t+1$. Then, it follows that

$$
n_{t+1}=(1-s) n_{t}+\mathcal{M}\left(u_{t}, v_{t}\right)=(1-s) n_{t}+q\left(\theta_{t}\right) v_{t} .
$$

\footnotetext{
${ }^{3}$ In the decentralized equilibria, the match surplus is divided between the worker and the firm according to some bargaining protocol. Below we assume that the planner cares only about the match output $y$, and not the division of the match surplus.
} 
The number of people with jobs at the beginning of $t+1$ include the number of undissolved matches which were formed at the start of $t$ that survived onto the start of $t+1$ [given by $(1-s) n_{t}$ ] in addition to the new matches formed at $t$. The following lemma will be useful below.

Lemma 1 If $A>1$, either $q(\theta)>1$ or $\theta q(\theta)>1$ for all $\theta$.

Lemma 1 demonstrates that for the model to be valid, $A$ must lie between 0 and 1 . This restriction on the scale parameter $A$ is somewhat novel in that standard references in the search-matching literature do not impose this restriction.

A planner's problem can be outlined as follows. Assume that the planner chooses an allocation that maximizes the discounted value of output and leisure net of vacancy costs. The principal tensions are as follows. An extra vacancy adds a cost, makes it easier for unemployed workers to find jobs, but makes it harder for firms to find workers. Employed workers "lose" leisure utility. More output is produced if the extra vacancy creates more matches. The planner takes all this into account when choosing the number of vacancies. Following Ljungqvist and Sargent (2000; p. 578), the planner chooses $v_{t}$ and next period's employment level, $n_{t+1}$ by solving $(\mathrm{P})$ where $(\mathrm{P})$ is defined by

$$
\max _{\left\{v_{t}, n_{t+1}\right\}_{t=0}^{\infty}} \sum_{t=0}^{\infty} \beta^{t}\left[y n_{t}+z\left(1-n_{t}\right)-c v_{t}\right]
$$

subject to (2), given a $n_{0}$. The Lagrangian can be written as

$$
£=\sum_{t=0}^{\infty}\left\{\beta^{t}\left[y n_{t}+z\left(1-n_{t}\right)-c v_{t}\right]+\lambda_{t}\left[(1-s) n_{t}+q\left(\frac{v_{t}}{1-n_{t}}\right) \cdot v_{t}-n_{t+1}\right]\right\}
$$

where $\lambda_{t}$ is the Lagrange multiplier on (2). Then, the first-order conditions with respect to $v_{t}$ and $n_{t+1}$ for an interior solution are given by

$$
-\beta^{t} c+\lambda_{t}\left[q^{\prime}\left(\theta_{t}\right) \theta_{t}+q\left(\theta_{t}\right)\right]=0
$$

and

$$
-\lambda_{t}+\beta^{t+1}(y-z)+\lambda_{t+1}\left[(1-s)+q^{\prime}\left(\theta_{t+1}\right) \theta_{t+1}^{2}\right]=0
$$

In Appendix A, we show that (3) and (4) reduce to the following first-order difference equation in $\theta$ :

$$
a \theta_{t+1}^{\alpha}-b \theta_{t+1}=\theta_{t}^{\alpha}-d
$$

where

$$
a \equiv \beta(1-s) \in(0,1)
$$




$$
b \equiv A \alpha \beta \in(0,1)
$$

using Lemma 1, and

$$
d \equiv \frac{A(1-\alpha) \beta(y-z)}{c}>0 .
$$

Equation (5) is the law of motion for the index of labor market tightness in the economy under the planner's solution. Such a sequence for $\theta$ must additionally satisfy $q(\theta) \in(0,1)$, and $n<1$.

As is clear from (5), it is clearly convenient to study the backward dynamics as it is derived explicitly from the first order conditions more easily than the more standard forward dynamics; in fact, $\theta_{t}$ is easily described as a function of $\theta_{t+1}$ while the relationship of $\theta_{t+1}$ to $\theta_{t}$ is typically a correspondence. ${ }^{4}$

\section{Stationary and non-stationary solutions}

Given values of $n_{0}$ and $\theta_{0}$ (or $v_{0}$ ), eq. (5) completely characterizes the unique trajectory of $\theta$ and all other endogenous variables. ${ }^{5}$ In other words, the backwards dynamics of this model can be characterized by the continuous four-parameter family of maps $g:\left[0, \theta_{\max }\right] \rightarrow\left[0, g_{\max }\right]$, where

$$
g(\theta)=\left(a \theta^{\alpha}-b \theta+d\right)^{\frac{1}{\alpha}}, \quad(\alpha, a, b, d) \in(0,1) \times(0,1) \times(0,1) \times(0, \infty),
$$

and $\theta_{\max }=\left(\frac{\alpha a}{b}\right)^{\frac{1}{1-\alpha}}$ and $g_{\max }$ is implicitly defined as the lowest positive root of the following equation:

$$
a g_{\max }^{\alpha}-b g_{\max }+d=0 .
$$

The first derivative of the map can be calculated as

$$
g^{\prime}(\theta)=\left(a \theta^{\alpha}-b \theta+d\right)^{\frac{1-\alpha}{\alpha}}\left(a \theta^{\alpha-1}-\frac{b}{\alpha}\right), \quad \theta \in\left[0, \theta_{\max }\right]
$$

which implies that $g$ is unimodal with a unique maximum at $\theta_{\max } \equiv\left(\frac{b}{a \alpha}\right)^{\frac{1}{\alpha-1}}$.

Lemma 2 The map $g(\theta)$ has a unique stationary solution $\left(\theta_{\text {ss }}\right)$ implicitly defined by

$$
a \theta_{s s}^{\alpha}-b \theta_{s s}=\theta_{s s}^{\alpha}-d .
$$

\footnotetext{
${ }^{4}$ Analysis of such backward difference equations are common. See for example, Grandmont (1985) and Michener and Ravikumar (1998) for analysis of perfect foresight backward dynamics in the overlapping generations model and a representative agent cash-in-advance model respectively.

${ }^{5}$ Ljungqvist and Sargent (2000; p. 578) assume that the planner just knows $n_{0}$. However, only if $\theta_{0}$ is additionally known can we compute the entire unique solution sequence $\left\{\theta_{t}\right\}_{t=1}^{\infty}$. From there, using (2), it is then possible to compute the optimal sequences $\left\{v_{t}\right\}_{t=0}^{\infty},\left\{u_{t}\right\}_{t=1}^{\infty}$, and $\left\{n_{t}\right\}_{t=1}^{\infty}$. To compute the steady state $\theta_{s s}$, however, information on $\theta_{0}$ is not needed.
} 
Note that it is not possible to obtain a closed form expression for $\theta_{s s}$. Below, we prove that $\theta_{s s}$ is unstable.

Proposition $1 \theta_{s s}$ is asymptotically stable in the non-standard backward dynamics implying it is unstable in the usual forward dynamics.

The implication is clear and powerful. The only stationary solution to the planner's problem is dynamically unstable in that it cannot be approached from any given set of initial conditions. The only way the planner can choose the stationary solution is to pick the initial conditions in such a way as to place the economy directly and immediately on the steady state. Hence, there is only one solution to the planner's problem and it is the stationary solution.

This result, at the very least, serves to expose the dynamic fragility of the planning solution to the discrete-time adaptation of the standard Pissarides (2001) model. For instance, the famed Hosios condition, a comparison of the social optimum to the market outcome, is always done at the steady state, which we have now shown is dynamically unstable.

Finally, we prove that the planning solution may exhibit oscillatory behavior but never any cycles.

\section{Proposition 2}

$$
-1<g^{\prime}\left(\theta_{s s}\right)<1
$$

It follows from Proposition 2 that since $g^{\prime}\left(\theta_{s s}\right)<0$ is possible, the planning solution may exhibit oscillatory behavior; however, since the oscillations are damped in the backward dynamics, they are necessarily undamped in the usual forward dynamics. Furthermore, since $g^{\prime}\left(\theta_{s s}\right)$ can never equal -1 or go below -1 , possibility of two or higher period cycles are ruled out.

\section{Concluding remarks}

This paper studies the planning solution to the standard Pissarides $(1990,2001)$ "textbook model" of search and matching in labor markets. We show that the planning solution is completely characterized by a first-order, non-linear scalar difference equation. There is a unique stationary solution. In fact, it is the only possible solution. The steady state is dynamically unapproachable from a arbitrary set of initial conditions. Additionally, the planning solution may exhibit oscillatory behavior but never any cycles. 


\section{Appendix}

\section{A Proof of Lemma 1}

Re-writing the conditions that $q(\theta)<1$ and $\theta q(\theta)<1$, we get

$$
q(\theta)=A \theta^{-\alpha}<1 \Leftrightarrow \theta>\left(\frac{1}{A}\right)^{-\frac{1}{\alpha}},
$$

and

$$
\theta q(\theta)=A \theta^{1-\alpha}<1 \Leftrightarrow \theta<\left(\frac{1}{A}\right)^{\frac{1}{1-\alpha}}
$$

That is, $\theta$ must lie in the interval $\left(\left(\frac{1}{A}\right)^{-\frac{1}{\alpha}},\left(\frac{1}{A}\right)^{\frac{1}{1-\alpha}}\right)$. For this interval to be non-empty, we require

$$
\begin{aligned}
\left(\frac{1}{A}\right)^{-\frac{1}{\alpha}} & <\left(\frac{1}{A}\right)^{\frac{1}{1-\alpha}} \Leftrightarrow 1<\left(\frac{1}{A}\right)^{\frac{1}{1-\alpha}+\frac{1}{\alpha}} \Leftrightarrow \\
1 & <\left(\frac{1}{A}\right) \Leftrightarrow A<1 .
\end{aligned}
$$

If $A$ is greater than one, it must therefore be the case that either $q(\theta)$ or $\theta q(\theta)$ is greater than 1.

\section{B Derivation of Equation (5)}

From (3), we obtain the following expression for $\lambda_{t}$ :

$$
\lambda_{t}=\frac{\beta^{t} c}{q^{\prime}\left(\theta_{t}\right) \theta_{t}+q\left(\theta_{t}\right)}
$$

Using (A1) for period $t$ and period $t+1$, we can insert this expression into (4):

$$
-\frac{\beta^{t} c}{q^{\prime}\left(\theta_{t}\right) \theta_{t}+q\left(\theta_{t}\right)}+\beta^{t+1}(y-z)+\frac{\beta^{t+1} c}{q^{\prime}\left(\theta_{t+1}\right) \theta_{t+1}+q\left(\theta_{t+1}\right)}\left[(1-s)+q^{\prime}\left(\theta_{t+1}\right) \theta_{t+1}^{2}\right]=0
$$

Inserting the expressions for $q(\theta)$ and $q^{\prime}(\theta)$ (recall that $q(\theta)=A \theta^{-\alpha}$ ) and re-arranging, we get:

$$
\beta(y-z)+\frac{\beta c}{-\alpha A \theta_{t+1}^{-\alpha}+A \theta_{t+1}^{-\alpha}}\left[(1-s)-\alpha A \theta_{t+1}^{-\alpha+1}\right]=\frac{c}{-\alpha A \theta_{t}^{-\alpha}+A \theta_{t}^{-\alpha}}
$$

From here, straightforward manipulation yields

$$
(1-s) \beta \theta_{t+1}^{\alpha}-\alpha A \beta \theta_{t+1}=\theta_{t}^{\alpha}-\frac{(1-\alpha) A \beta(y-z)}{c},
$$

which immediately provides the desired expression in (5). 


\section{Proof of Lemma 2}

Re-writing the equation which defines the steady state, we get:

$$
(a-1) \theta_{s s}^{\alpha}+d=b \theta_{s s}
$$

First note that the function on the left hand side of (A3) takes the value $d>0$ when $\theta=0$, while the right hand side of (A3) starts out at 0 . Further more the left hand side is strictly decreasing, as $a<1$, and the righthand side is strictly increasing as $b>0$. Therefore the map $g(\theta)$ has a unique stationary solution

\section{Proof of Propositions 1 and 2.}

Note that if $g^{\prime}\left(\theta_{s s}\right) \in(-1,1)$, the map is stable in the backwards dynamics, and therefore it is unstable in the forward dynamics. Therefore the proof of both propositions will be complete if we can show that $g^{\prime}\left(\theta_{s s}\right) \in(-1,1)$. Using the definition of $\theta_{s s}$, namely that $\theta_{s s}=\left(a \theta_{s s}^{\alpha}-b \theta_{s s}+d\right)^{\frac{1}{\alpha}}$, as well as the definition of the parameters, we get the following expression for $g^{\prime}\left(\theta_{s s}\right)$ :

$$
\begin{aligned}
g^{\prime}\left(\theta_{s s}\right) & =\left(a \theta_{s s}^{\alpha}-b \theta_{s s}+d\right)^{\frac{1-\alpha}{\alpha}}\left(a \theta_{s s}^{\alpha-1}-\frac{b}{\alpha}\right)=\theta_{s s}^{1-\alpha}\left(a \theta_{s s}^{\alpha-1}-\frac{b}{\alpha}\right) \\
& =\left(a-\frac{b}{\alpha} \theta_{s s}^{1-\alpha}\right)=\beta(1-s)-A \beta \theta_{s s}^{1-\alpha}=\beta\left(1-s-q\left(\theta_{s s}\right)\right)
\end{aligned}
$$

Since $0<\beta<1,0<s<1$, and $0<q(\theta)<1$, it is immediately clear that $g^{\prime}\left(\theta_{s s}\right) \in(-1,1)$ 


\section{References}

[1] Andalfatto, D, 1996. Business cycles and labor market search. American Economic Review, 86(1), $112-32$.

[2] Cole H.L., Rogerson, R., 1999. Can the Mortensen-Pissarides matching model match the businesscycle facts? International Economic Review, 40(4), 933-59.

[3] Cooley, T.F., Quadrini, V., 1999. A neoclassical model of the Phillips curve relation. Journal of Monetary Economics, 44 (2), 165-193.

[4] Diamond, P.A. 1982. Aggregate demand management in search equilibrium. Journal of Political Economy 90, 881-94

[5] Diamond, P.A., Fudenberg, D. 1989. Rational expectations business cycles in search equilibrium. Journal of Political Economy 97, 606-619.

[6] Drazen, A., 1988. Self-fulfilling optimism in a trade-friction model of the business cycle. American Economic Review, 78(2), 369-72.

[7] Garibaldi, P., Wasmer, E. 2001. Labor market flows and equilibrium search unemployment. Institute for the Study of Labor, Bonn, Discussion Paper No. 406.

[8] Grandmont, J.-M., 1985. On endogenous competitive business cycles. Econometrica 53, 995-1045.

[9] Ljungqvist, L. and Sargent, T. 2001 Recursive Macroeconomic Theory. MIT Press, Cambridge Massachusetts

[10] Medio., A. 1998. Nonlinear dynamics and chaos. part I: a geometrical approach. Macroeconomic Dynamics, Vol 2 (4)

[11] Merz, M. 1995. Search in the labor market and the real business cycle. Journal of Monetary Economics, 36(2), 269-300.

[12] Mortensen, D. 1999. Equilibrium unemployment cycles. International Economic Review, 40, 889914

[13] Michener, R. and Ravikumar, B. 1998 Chaotic dynamics in a cash-in-advance economy, Journal of Economic Dynamics and Control, 22 (7), 1117-1137.

[14] Pissarides, C.A. 1990. Equilibrium Unemployment Theory, Oxford: Blackwell.

[15] — 2001 Equilibrium Unemployment Theory, MIT Press

[16] Shi, S., Quan W., 1999. Labor market search and the dynamic effects of taxes and subsidies. Journal of Monetary Economics, 43 (2), 457-495.

[17] Shimer, R and L. Smith 2001 Non-stationary search. mimeo Princeton University

[18] Yashiv E. 2000. The determinants of equilibrium unemployment. American Economic Review, 90(5), 1297-1322.

[19] Yuan, M., Li, W. 2000. Dynamic employment and hours effects of government spending shocks. Journal of Economic Dynamics and Control, 24 (8), 1233-1263. 